\title{
Comparison of the Influence of Drag Models in CFD Simulation of Particle Mixing and Segregation in a Rotating Cylinder
}

\author{
Sumudu S. Karunarathne Lars-Andre Tokheim
}

Department of Process, Energy and Environmental Technology, University College of Southeast Norway,

\{sumudu.karunarathne, lars.a.tokheim\} @usn. no

\begin{abstract}
CFD modelling was used to simulate particle segregation in a transverse plane of a rotating cylinder under different particle-particle drag models. The Eulerian method was used to model the dense particulate phases in the system. Two types of particles, with different density and size, were used in the study. The simulations were performed under the rolling mode since this mode is believed to give good particle-particle mixing. The drag models of Schiller-Naumann, MorsiAlexander and Syamlal-O'Brien Symmetric were applied in the modelling of particle-particle drag and results were compared with experiments. All the drag models were able to model the particle segregation. The Schiller-Naumann model and the Morsi-Alexander model showed good agreement with the experimental results while the Syamlal-O'Brien-Symmetric model had some deviations.
\end{abstract}

Keywords: particle segregation, rotating cylinder drag models, CFD

\section{Introduction}

Particulate materials are used in various types of industrial processes. Mixing is a physical process in which several granular phases are mixed together with the aim to make a homogeneous particle mixture. Rotating cylinders are widely used process units in many industrial applications, such as in cement, mineral and pharmaceutical industries. However, unlike in liquids, mixing time does not always improve the degree of particle mixing. The particle motion in the bed may give mixing of the different particle phases, but it may also give segregation (Karunarathne et al, 2016), as indicated in Figure 1. Consequently, it is vital to have a better understanding of factors that may cause particle segregation in such units.

When simulating mixing of particulate phases, it is quite common to apply the Euler-Lagrange method. The Discrete Element Method (DEM) is a Lagrangian approach (Yamada et al, 2011) that uses laws of motion (Newton's second law) in simulation to provide dynamic information about particles in motion (Yang et $a l, 2003)$. It allows studying micro-dynamics of powder flows that is difficult measure through experiments. DEM has been used in simulations of ball mills, rotary vessel mixtures, bin-hopper systems and several other applications, as DEM simulations can generate accurate results on granular behavior (Yamada et al, 2011). The disadvantage of DEM is that it is computationally intensive when the particle size becomes small (Soni et $a l, 2016)$. In such cases, the Euler-Euler method may be useful. In this method, both fluid and solid phases are treated as interpenetrating continua and show the flow dynamics by using averaged equations of motion. Compared to the Euler-Lagrange approach, the EulerEuler approach is often preferred due to the less computational demand (Mazzei, 2008).

In the current study, the Euler-Euler method was applied to model the particulate phases, which are quite dense. In this method, the particle bed is considered as a continuum, and granular kinetic theory is used to evaluate the viscosity terms in the transport equation. The drag force describes the momentum exchange between phases in the system. This interaction can be described through a set of mathematical formulations that apply to different conditions.

In a rotating cylinder, two types of drag forces are present and must be considered in CFD modelling of a binary solid mixture. In Fluent, several drag models can be selected to calculate the fluid-fluid drag, the particlefluid drag and the particle-particle drag.

This study was performed to better understand the behavior of particle mixing and segregation in a binary mixture in a rotating drum. The calculation problem was simplified to a two-dimensional transverse plane, and the CFD simulations were done with the commercial software Fluent, version 16.2. Three different models the drag between particles in two phases were compared. The Schiller-Naumann (Fluent, 2013) model is a general model for fluids; the Morsi-Alexander model covers a large range of Reynolds numbers (Morsi and Alexander, 1972); the Syamlal-O'Brien-Symmetric model is specifically derived for a pair of solid phases (Syamlal, 1987). The simulation results were compared with lab experiments to identify which model that gives the best fit with experimental results.

\section{Theory}

There are two approaches that can be applied in CFD simulations of particles in a rotating cylinder: In the Lagrange method, Newton's second law is used to 
calculate the particle velocities and their trajectories (Fluent, 2013). This is a good approach for dilute particle phases. If the particle phase is dense, it can be modelled as a continuum using the Eulerian method. The flow properties of a granular phase can be evaluated using granular kinetic theory in that the term called granular temperature is considered to be proportional to the square of the velocity fluctuations in the solid phase. This is an analogy to the thermodynamic temperature of gases (Benzarti et al, 2012).

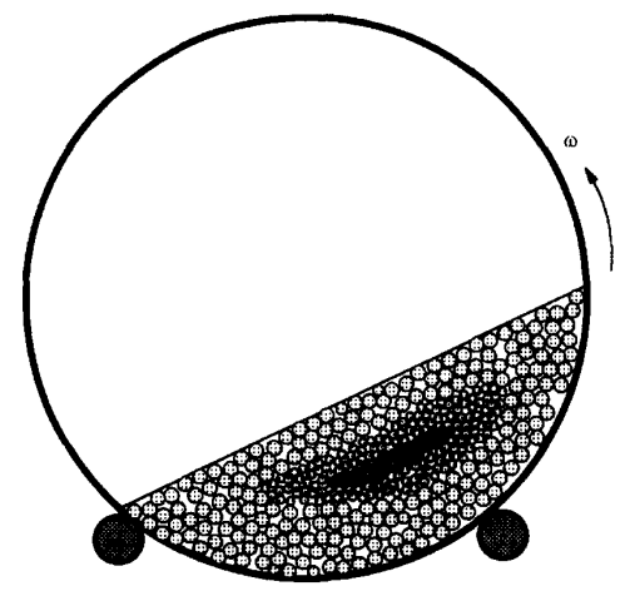

Figure 1. Schematic of radial segregation of particles of different size in a rotary kiln (Boateng and Barr, 1996)

A drag model gives valuable information about the momentum exchange between the phases in a multiphase flow situation. Several articles have focused on finding the optimum drag model for various engineering applications. Benzarti et al, (2012) performed a numerical parametric study to model the fluid behavior in the riser of a bubbling fluidized bed (BFB). The gas-solids drag was modelled and compared using three drag models: Gidaspow, Syamlal and O'Brien and EMMS. A similar type of work has been done by Lundberg and Halvorsen, (2008), who also implemented the Richardson-Zaki drag model, the RUC drag model and the Hill-Koch-Ladd drag model in the Fluent environment. Silva et al, (2015) studied the particle distribution in a highly concentrated particleliquid stream in a pipe using a mixture model. The drag models of Schiller-Naumann and Gidaspow-ShillerNaumann were used to model the drag force between the particles and the fluid. For a solids-liquid suspension with a high particle concentration at intermediate velocities, the Schiller-Naumann drag correlation showed the best agreement.

A detailed description of the Eulerian method is given in a previous paper by Karunarathne et al, (2016) and is not repeated here. Instead, the focus is put on discussing the drag models used in this study.

\subsection{Drag models for fluid flows}

The exchange coefficient for bubbly liquid-liquid mixtures or bubbly gas-liquid mixtures can be written as (Fluent, 2013):

$$
\begin{aligned}
& k_{p q}=\frac{\alpha_{p} \alpha_{q} \rho_{p} f}{\tau_{p}} \\
& \tau_{p}=\frac{\rho_{p} d_{p}^{2}}{18 \mu_{q}}
\end{aligned}
$$

Different drag models apply different equations to represent the drag function $f$. Some drag models even use another representation of the exchange coefficient $k_{p g}$.

\subsubsection{Schiller-Naumann}

This model is used for modelling of drag between fluid phases in multiphase flow. The drag function $f$ is given as:

$$
f=\frac{C_{D} \operatorname{Re}}{24}
$$

The drag coefficient $C_{D}$ is given as:

$$
C_{D}= \begin{cases}24\left(1+0.15 \mathrm{Re}^{0.687}\right) / \mathrm{Re} & \mathrm{Re} \leq 1000 \\ 0.44 & \mathrm{Re} \geq 1000\end{cases}
$$

The relative Reynold number Re for the primary phase $q$ and secondary phase $p$ is given as:

$$
\operatorname{Re}_{p q}=\frac{\rho_{q}\left|\overrightarrow{v_{p}}-\overrightarrow{v_{q}}\right| d_{p}}{\mu_{q}}
$$

Then the relative Reynolds number for the secondary phases $p$ and $r$ is:

$$
\operatorname{Re}_{r p}=\frac{\rho_{r p}\left|\overrightarrow{v_{r}}-\overrightarrow{v_{q}}\right| d_{r p}}{\mu_{r p}}
$$

The mixture viscosity of phase $p$ and $r$ is:

$$
\mu_{r p}=\alpha_{r} \mu_{r}+\alpha_{p} \mu_{p}
$$

\subsubsection{Morsi-Alexander}

The drag function $f$ in the Morsi-alexander model is:

$$
f=\frac{C_{D} \operatorname{Re}}{24}
$$

The drag coefficient is:

$$
C_{D}=a_{1}+\frac{a_{2}}{\operatorname{Re}}+\frac{a_{3}}{\operatorname{Re}^{2}}
$$


$a$ is defined as:

$$
a_{1}, a_{2}, a_{3}= \begin{cases}0,24,0 & 0<\operatorname{Re}<0.1 \\ 3.690,22.73,0.0903 & 0.1<\operatorname{Re}<1 \\ 1.222,29.1667,-3.8889 & 1<\operatorname{Re}<10 \\ 0.6167,46.50,-116.67 & 10<\operatorname{Re}<100 \\ 0.3644,98.33,-2778 & 100<\operatorname{Re}<1000 \\ 0.357,148.62,-47500 & 1000<\operatorname{Re}<5000 \\ 0.46,-490.546,578700 & 5000<\operatorname{Re}<10000 \\ 0.5191,-1662.5,5416700 & \operatorname{Re}>10000\end{cases}
$$

\subsubsection{Syamlal-O'Brien Symmetric}

This model is applied for drag between particulate phases. The solid-solid exchange coefficient $k_{l s}$ is given as:

$$
k_{l s}=\frac{3\left(1-e_{l s}\right)\left(\frac{\pi}{2}+C_{f r, l s} \frac{\pi^{2}}{8}\right) \alpha_{s} \rho_{s} \alpha_{l} \rho_{l}\left(d_{l}+d_{s}\right)^{2} g_{0, l s}}{2 \pi\left(\rho_{l} d_{l}^{3}+\rho_{s} d_{s}^{3}\right)}\left|\overrightarrow{v_{l}}-\overrightarrow{v_{s}}\right|(11)
$$

\section{Method}

\subsection{Simulation setup}

The geometry was represented by a two-dimensional transverse plane with a diameter of $0.19 \mathrm{~m}$, which was also the size of the experimental setup, and a mesh with 3500 elements was created.

The transverse plane was set to rotate around its axis, and a no-slip condition was applied between the granular material and the wall, i.e. the relative velocity between the particles and the wall is considered to be zero.

The fluids in the system were considered as incompressible and model equations for the Eulerian approach were solved using a pressure-based solver with the finite volume method. The pressure-velocity coupling was done by the SIMPLE algorithm (Patankar and Spalding, 1972). The transport equations were discretized according to the second order upwind scheme, and the QUICK scheme was used to discretize volume fraction of the phases (Versteeg and Malalasekera, 2007). The time step of the simulation was set to $10^{-3} \mathrm{~s}$, and the residual values for convergence were set to $10^{-3}$.

The simulations were performed with three different drag models to investigate the effect of particle-particle drag forces on mixing and segregation. These simulations were carried out with four different cylinder filling degrees: $10,15,20$ and $25 \%$. The rotational velocity of the transverse plane was set to $3 \mathrm{rpm}$ in all simulations to maintain the motion of the particle bed under rolling mode.

\subsection{Experimental setup}

Mixing of two particular phases was tested in lab experiments using a rotating drum made of Lexan, 19 $\mathrm{cm}$ in diameter and with a length of $29 \mathrm{~cm}$. Two types of spherical particles with different densities and diameters were used in the experiments, see Table 1. The particles were coloured yellow (A) and blue (B) to make the mixing and segregation pattern more visible.

Microscopy and sieving analysis were used to determine the particle diameters, and the true density of the particles was measured using an autopycnometer of model 1320 from Micromeritics. Figure 2 illustrates particle filling through a transverse plane; initially the particles were arranged as separate layers stacked on top of each other. As in the simulations, the rotational velocity of the drum was set to $3 \mathrm{rpm}$ in all experiments. In order to compare results with the simulations, all experiments were done at particle filling degrees of 15 and $25 \%$ in order to observe its influence on mixing and segregation.

Table 1. Particle properties

\begin{tabular}{|l|c|c|}
\hline Description & Symbol & Value \\
\hline Particle A density & $\rho_{A}$ & $2537 \mathrm{~kg} / \mathrm{m}^{3}$ \\
\hline Particle B density & $\rho_{B}$ & $2417 \mathrm{~kg} / \mathrm{m}^{3}$ \\
\hline Particle A size & $d_{A}$ & $1500 \mu \mathrm{m}$ \\
\hline Particle B size & $d_{B}$ & $3000 \mu \mathrm{m}$ \\
\hline Particle A colour & - & Yellow \\
\hline Particle B colour & - & Blue \\
\hline
\end{tabular}

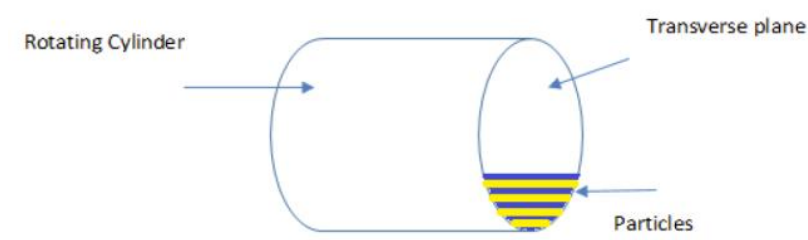

Figure 2. View of the particle filling through a transverse plane in the rotating cylinder

\section{Results}

The experimental results show that the particles segregate when the cylinder rotates around its main axis. This segregation, which is seen in Figures 3-6, is mainly due to the variation of particle diameter in the two different particle phases. Particle dynamics in the active layer of the rolling mode allow finer particles to concentrate at the mid-chord section (Boateng and Barr, 1996). Subsequently, fine particles move through the voids between bigger particles under the action of gravity and this is called percolation. As a result, fine particles accumulate at the middle of the particle bed. 
The Euler-Euler method was able to simulate the particle mixing in the transverse plane of a rotating cylinder under different drag models. The simulations showed that particles with a small diameter tend to concentrate at the middle of the particle bed in the transverse plane. At the same time, particles with a larger diameter tend to move to the bottom of the particle bed.

Figures 3-6 show compare the simulated results from the three drag models and the results obtained from experiments. The volume fractions of both particulate phases were calculated in all simulation cases. Considering the particle segregation, the simulations indicate that the Schiller-Naumann and MorsiAlexander drag models are in better agreement with the experimental results. Both these models were able to simulate the segregation behavior observed in the experiments. The results obtained through the use of the Syamlal-O'Brien Symmetric model deviate from the results of the other two drag models. Although the model was able to simulate some segregation, it did not show the complete separation of the two particulate phases which was found by applying the other two models and in the experiments.

In rolling mode, particles in the passive layer do not experience much particle collision when they move. The Syamlal-O'Brien Symmetric model is often used in CFD simulations of fluidized beds, and it gives good results as those systems involve a high rate of collision among the particles. The Schiller-Naumann and MorsiAlexander models are, however, more appropriate to model the drag between continua (as opposed to particles), and in the current simulation, the particulate phases are modelled as continua.

In order to examine the effect of degree of particle filling on mixing and segregation, the experiments were done at two different degrees of filling under rolling mode. There was, however, no significant improvement in particle mixing after increasing the particle bed height from 15 to $25 \%$. The simulations confirmed this observation, see Figures 3-6.

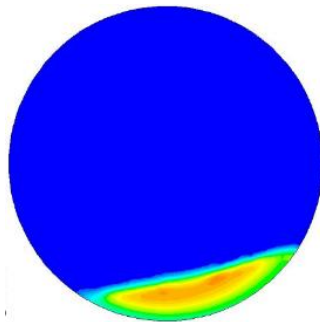

a

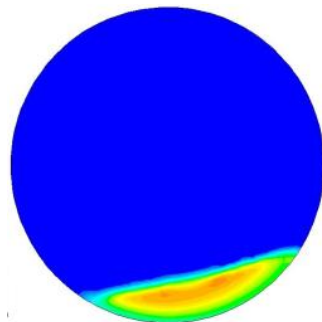

b

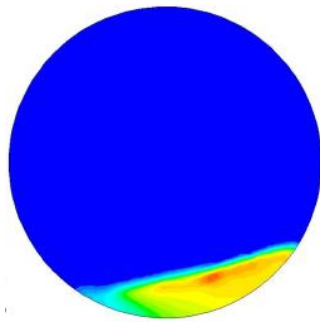

C

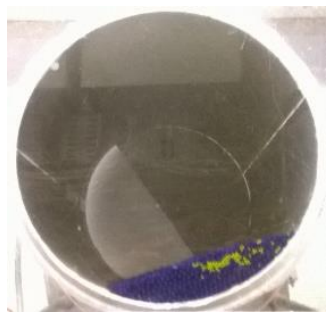

d

Figure 3. Volume fraction of "A" particles at $15 \%$ of degrees of particle filling (a: Schiller-Naumann, b: MorsiAlexander, c: Syamlal-O’Brien-Symmetric, d: Experiment). Yellow (A) and Blue (B) particles

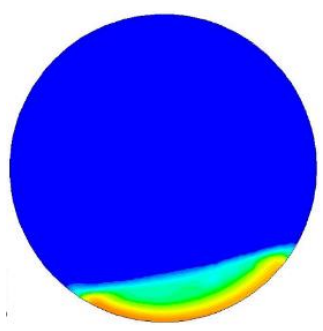

a

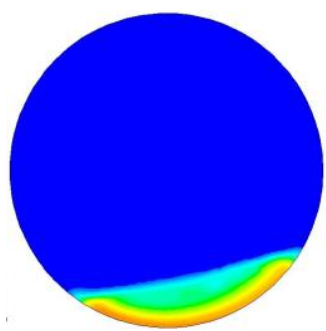

b

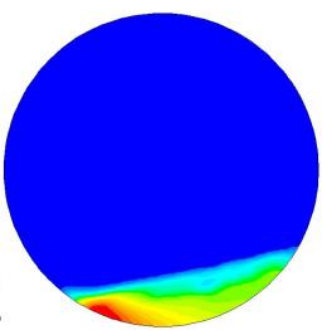

c

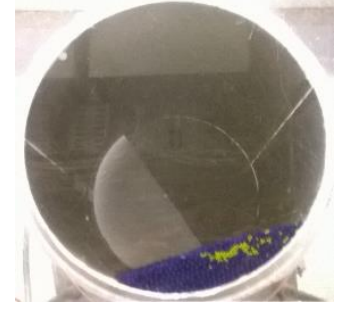

d

Figure 4. Volume fraction of "B" particles at $15 \%$ of degrees of particle filling (a: Schiller-Naumann, b: MorsiAlexander, c: Syamlal-O'Brien-Symmetric, d: Experiment). Yellow (A) and Blue (B) particles 


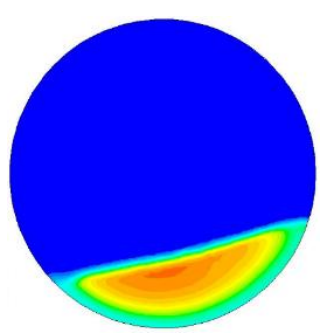

a

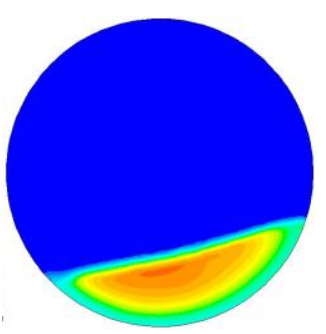

$\mathrm{b}$

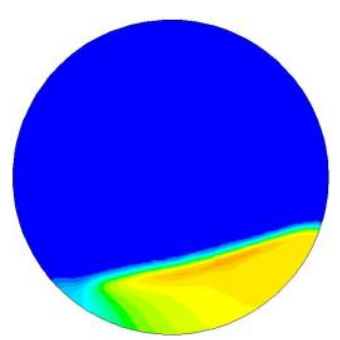

C

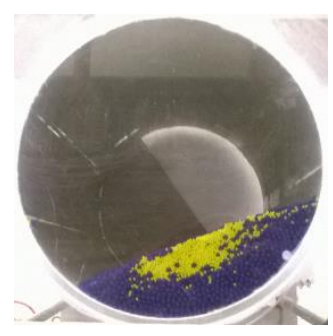

d

Figure 5. Volume fraction of "A" particles at $25 \%$ of degrees of particle filling (a: Schiller-Naumann, b: MorsiAlexander, c: Syamlal-O’Brien-Symmetric, d: Experiment). Yellow (A) and Blue (B) particles

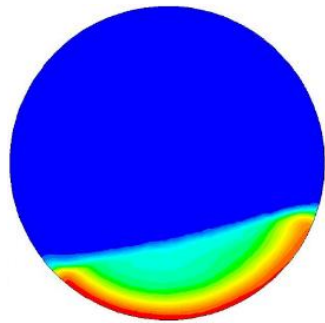

a

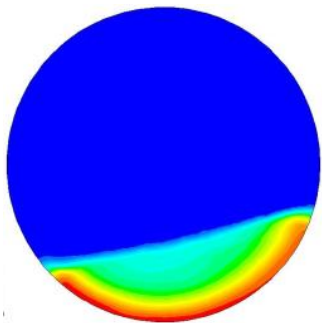

b

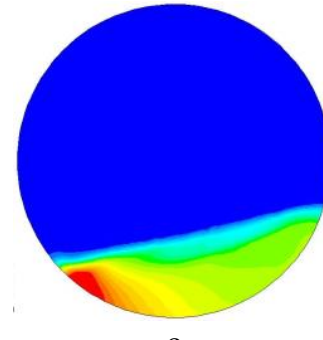

$\mathrm{c}$

Figure 6. Volume fraction of "A" particles at $25 \%$ of degrees of particle filling (a: Schiller-Naumann, b: MorsiAlexander, c: Syamlal-O’Brien-Symmetric, d: Experiment). Yellow (A) and Blue (B) particles

\section{Conclusion}

The Euler-Euler approach was able to simulate the particle dynamics in a transverse plane of a rotating cylinder applying different drag models. The particleparticle drag model has a significant influence on the mixing and segregation of particles in a transverse rotating plane.

The predicted particle distribution patterns from applying the Schiller-Naumann and Morsi- Alexander drag models under rolling mode were in better agreement with the experimental results than those obtained by using the Syamlal-O'Brien Symmetric drag model.

The increase of degree of particle filling did not show any significant effect on the mixing behavior. This was seen in simulations as well as in experiments.

\section{Nomenclatere}

$\begin{array}{ll}C_{D} & \text { Drag coefficient [-] } \\ C_{f r} & \text { Coefficient of friction [-] } \\ d & \text { Diameter [m] } \\ e & \text { Coefficient of restitution [-] } \\ f & \text { Drag function [-] } \\ g_{0} & \text { Radial distribution [-] }\end{array}$

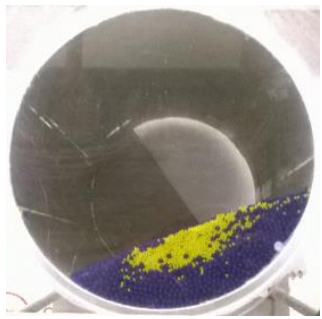

d

\author{
Exchange-coefficient $\left[\mathrm{kg} /\left(\mathrm{m}^{3} \cdot \mathrm{s}\right)\right]$ \\ Velocity $[\mathrm{m} / \mathrm{s}]$ \\ Reynolds number [-] \\ Volume fraction [-] \\ Viscosity $[\mathrm{Pa} \cdot \mathrm{s}]$ \\ Density $\left[\mathrm{kg} / \mathrm{m}^{3}\right]$ \\ Particulate relaxation time [s]
}

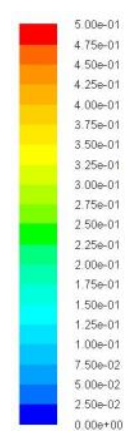

$v$

$\alpha$

$\mu$

$\rho$

$\tau$

\section{References}

A. A. Boateng and P.V. Barr. Modelling of particle mixing and segregation in the transverse plane of a rotary kiln. Chemical Engineering Science, 51: 4167-4181, 1996.

Fluent . ANSYS Fluent Theory Guide. Ansys Fluent, 2013.

H. K. Versteeg and W. Malalasekera. An introduction to computational fluid dynamics, England, Pearson Education Limited, 2007.

J. Lundberg and B. M. Halvorsen. A review of some exsisting drag models describing the interaction between phases in a bubbling fluidized bed. Proceedings of the 49th Conference on Simulation and Modelling, SIMS 49, October 7th-8th 2008 Oslo, Norway.

L. Mazzei. Eulerian modelling and computational fluid dynamics simulation of mono and polydisperse fluidized suspensions. Department of Chemical Engineering, University College London, 2008 
M. Syamlal. The particle-particle drag term in a multiparticle model of fluidization, 1987.

R. Silva, C. Cotas, P. Garcia, M. Faia and G. Rasteiro. Particle distribution studies in highly concentrated solid-liquid flows in pipe using the mixture model. The 7th world congress on particle technology (WCPT7). Procedia Engineering, 1016$1025,2015$.

R. K. Soni, R. Mohanty, S. Mohanty and B.K. Mishra. Numerical analysis of mixing of particles in drum mixers using DEM. Advanced Powder Technology, 27: 531-540, 2016.

R.Y. Yang, R.P. Zou and A.B. Yu. Microdynamic analysis of particle flow in a horizontal rotating drum. Powder Technology, 130: 138-146, 2003.

S. A. Morsi and A. J. Alexander. An investigation of particle trajectories in two-phase flow systems. Journal of Fluid Mechanics, 55: 193-208, 1972.

S. Benzarti, H. Mhiri and H. Bournot. Drag models for simulation gas-solid flow in the bubbling fluidized bed of fcc particles. International Journal of Chemical, Molecular, Nuclear, Materials and Metallurgical Engineering, 6 :111-116, 2012.

S. Karunarathne, C. Jayarathna and L.A. Tokheim. 2016. Impact of particle diameter, particle density and degree of filling on the flow behavior of solid particle mixtures in a rotating drum. 9th EUROSIM Congress on Modelling and Simulation, 2016.

S. Patankar and D. Spalding. A calculation procedure for heat, mass and momentum transfer in three-dimensional parabolic flows. International Journal of Heat and Mass Transfer, 15, 1972 .

Y. Yamada, M. Sakai, T. Nonoue and K. Takahashi. Development of a Lageangian-Lagrangian coupling method for solid-liquid flows. Chemeca 2011, Engineering a better world, September 18th-21st, NSW, Australia, 2831-2840, 2011. 